\title{
Meso-scale computer modeling of lipid-DNA complexes for gene therapy
}

\author{
Oded Farago, ${ }^{1}$ Niels Grønbech-Jensen, ${ }^{2}$ and Philip Pincus ${ }^{3,4}$ \\ ${ }^{1}$ Department of Biomedical Engineering, Ben Gurion University, Be'er Sheva 84105, Israel \\ ${ }^{2}$ Department of Applied Science, University of California, Davis, California 95616 \\ ${ }^{3}$ Materials Research Laboratory, University of California, Santa Barbara, California 93106 \\ ${ }^{4}$ Physics Department, Korea Advanced Institute of Science and Technology (KAIST), \\ 373-1 Kusong-dong, Yusong-gu, Taejon 305-701, South Korea.
}

\begin{abstract}
We report on a molecular simulation method which captures the self-assembly of cationic lipidDNA (CL-DNA) gene delivery complexes. Computational efficiency required for large length- and time-scale simulations is achieved through a coarse-grained representation of the intra-molecular details, and via inter-molecular potentials, which effectively mimic the hydrophobic effect without explicit solvent. In addition to showing spontaneous self-assembly of complexes, the broad utility of the model is illustrated by demonstrating excellent agreement with X-ray diffraction experimental data for the dependence of the spacing between DNA chains on the concentration of CLs. At high concentrations, the large electrostatic pressure induce the formation of pores in the membranes through which the DNA molecules may escape the complex. We relate this observation to the origin of recently observed enhanced transfection efficiency of lamellar CL-DNA complexes at high charge densities.
\end{abstract}

PACS numbers:

Modeling and simulation of biological systems possess significant challenges due to the spatial complexity of such systems and by the accompanying range of temporal scales involved. In the case of lipid bilayers, for instance, the width of the bilayer is in the nanometer range, whereas the lateral dimension may extend up to several micrometers. The corresponding time scales span an even larger range of orders of magnitude. To address the multi-scale nature of membranes, a variety of models have been devised which differ in the length- and timescale of the phenomena of interest [1]. The two most extreme approaches are: (1) atomistic computer simulations in which the lipids and the embedding solvent are modeled explicitly in full detail 2], and (2) phenomenological models such as the effective Helfrich Hamiltonian where the bilayer membrane is treated as a smooth continuous surface with a small number of elastic coefficients [3].

The gap between those two approaches can be bridged by coarse-grained (CG) computer models in which groups of several atoms are represented by unified particles interacting via effective potentials [4]. The essential benefit of such models is that they have condensed the most detailed variations in both time and space, while retaining the fundamental properties of membrane self-assembly and elasticity. The level of detail employed by different CG models varies greatly. Some CG models are constructed to mimic specific lipidic systems by selecting simplified representations for water molecules and different chemical groups which constitute the lipids, and by developing effective force-fields that reproduce key structural features known from experiments and atomistic simulations [5]. In coarser-grained models the amphiphilic molecules are constructed from only two types of particles - hydrophilic and hydrophobic - which, respectively, are attracted and repelled from a third kind of particles representing the solvent [ $[$ ] . Such models address more general features of self-assembly rather than specific systems.

More recently, a number of research groups have developed CG models in which the bilayer membranes are simulated without direct representation of an embedding solvent; this is accomplished by constructing intermolecular force fields that mimic effects of hydration 6 , 8, 9, 10]. The development of these "water-free" models is an important accomplishment in large-scale membrane simulations, considering the fact that the number of solvent particles in conventional CG models is significantly larger than the number of lipids. Most of these models employ simple Lennard-Jones (LJ) type pair-potentials [8, 9, 10], and exhibit spontaneous self-assembly to a membrane as demonstrated in Fig. 10 (A).

The computational simplicity and efficiency of waterfree CG models make them excellent tools for testing mesoscopic scale theories of membranes and gaining insight into the basic molecular mechanisms that govern these systems. More importantly, these models may serve as platforms for CG simulations of more complex systems including bio-polymers and networks. This paper reports on such a computational study of complexes of cationic lipid and DNA (CL-DNA) in the context of recent experiments on molecular assemblies. The molecular assemblies are formed spontaneously when DNA is mixed with cationic and neutral lipids in an aqueous environment [1, 12]. Their formation is driven by the electrostatic attraction between the negatively charged DNA and the cationic lipid head-groups, and by the entropic gain associated with the concurrent release of the tightly bound counterions from the CL and the DNA [11, 12, 13]. Xray diffraction experiments have revealed that CL-DNA complexes exist in a variety of mesoscopic structures [14], including a lamellar phase where the DNA is intercalated 
A

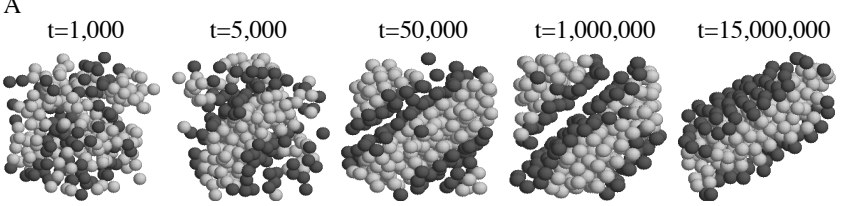

B

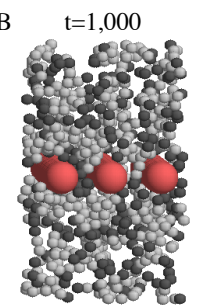

$t=2,000,000$
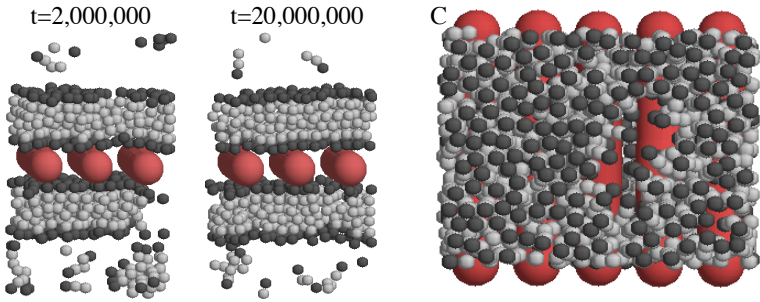

FIG. 1: (A) Self-assembly of a bilayer patch of 100 lipids. Lipids are modeled as trimers where the black and grey spheres represent hydrophilic and hydrophobic particles, respectively (see details in Refs. [8]). Each Monte Carlo time step includes (on average) an attempt to move and rotate each molecule. The membrane in the last figure has been rotated for a better viewing of its structure. (B) Self-assembly of a CL-DNA complex consisting of a total number of 240 lipids (of which 90 are charged). Red rods represent DNA segments. (C) Equilibrium configuration of a complex with $\phi_{c} \sim 0.9$ whose membranes develop pores.

in between lipid bilayers 11]. The DNA strands form a one-dimensional (1D) ordered array, where the DNA interaxial spacing $d_{\mathrm{DNA}}$ decreases with the charge density of the membranes. Isoelectric complexes, where the charges on the DNA exactly match those on the CL, are the most stable ones since they enable nearly complete counterion release [13].

CL-DNA complexes have attracted much attention because of their potential use as non-viral transfection vectors in gene therapy [14, 15, 16, 17]. Cationic liposome transfer vectors exhibit low toxicity, nonimmunogenicity, and ease of production 15, 18, but their transfection efficiency (TE) remains low compared to that of viral vectors [19, 20]. This has spurred an intense research activity aimed at enhancing TE [16, 19, 20]. Recognizing that the structure of CL-DNA complexes may strongly influence their function and TE, much of the effort in theoretical and experimental studies has been devoted to understanding the mechanisms governing complex formation, structure, and phase behavior [13, 14, 21, 22, 23].

To the best of our knowledge, only a single attempt has so far been made to study CL-DNA complexes using molecular simulations 24]. That study employed a fully atomistic description of both the lipids and the
DNA and has, consequently, been limited to a small system consisting of 48 lipids (of which 20 were charged) and a short DNA segment of 10 base pairs. The duration of the simulations was of several nanoseconds. The mesoscopic regime encompassing the statistics and evolution of large molecular ensembles is, however, inaccessible to full atomistic simulations due to the computational requirements, and only continuum behavior of existing CL-DNA structures can be addressed based on free energy functionals which are insensitive to the fine details of the lipids and DNA 21]. Thus, our understanding of many important CL-DNA complex features, such as the process of self-assembly and membrane evolution, mesoscopic structure and defects, can be addressed only through CG simulations.

The model presented here is based on the CG waterfree membrane model, which produces self-assembled bilayer membranes such as the one depicted in Fig. 1(A); model details are given in Refs. [8]. The lipids consist of one hydrophilic (representing the head group) and two hydrophobic (representing the tail) spherical particles with short-range pair-interactions between them. We set the diameter of these particles $\sigma \simeq 6.3 \AA$ (see the definition of $\sigma$ in Ref. [8]). For this value of $\sigma$ the area per lipid in the original model $a_{\text {lipid }} \simeq 70 \AA^{2}$. In the present model a fraction $\phi_{c}$ of the hydrophilic head groups carry charge $+e$. DNA is modeled as a rigid rod with a uniform axial charge density $\lambda_{\mathrm{DNA}}=-e / 1.7 \AA$ and radius $R_{\mathrm{DNA}}=10 \AA$. Excluded volume interactions between rods $(\mathrm{R})$ and spheres $(\mathrm{S})$ are introduced via a truncated and shifted potential of the form: $U_{R S} / k_{B} T=$ $50\left\{\left[\left(\sigma / 2+R_{\mathrm{DNA}}\right) / r\right]^{12}-1\right\}$, where $r$ is the distance between the center of the sphere and the axis of symmetry of the rod. The distance between nearest-neighbor rods is restricted to $d_{\mathrm{DNA}} \geq 2 R_{\mathrm{DNA}}$.

We study isoelectric complexes where the total charges of the DNA and the CLs neutralize each other, with no added counterions. Simulations of the quasi twodimensional (2D) complex are conducted in a rectangular system of size $L_{x} \times L_{y} \times L_{z}$, with full periodic boundaries along the $x$ and $y$ directions [25], and periodicity with respect to only lipid mobility and short-range interactions in the $z$ direction. Simulations were performed at room temperature and with a bulk water uniform dielectric constant $\epsilon_{r}=78$. The rods are arranged in a one-dimensional array with equal spacing in the $x y$-plane along the $y$-direction. We first examined the ability of the complex to self-assemble spontaneously: 240 lipids, of which 90 are charged, were randomly distributed in a box of size $L_{x}=94 \AA, L_{y}=51 \AA, L_{z}=157 \AA$. Three equally-spaced DNA strands, each carrying a total charge $-30 e$, were placed at the mid-plane $z=L_{z} / 2$ of the box. Canonical ensemble Monte Carlo (MC) simulations were used to generate the temporal evolution of the lipids, while the positions of the rods were fixed. Each MC step consists of (on average) an attempt to translate (and make some minute changes in the relative locations of the three particles with respect to each other) and rotate each 
lipid. Typical evolution of the system is depicted in Fig. 1 (B). The Figure demonstrates that a complex is formed quite rapidly, but full association of the lipids is much slower and has not been accomplished by the end of the MC run. The large majority of CLs (except for, typically, as few as 3-4 molecules) tend to reside in the two inner monolayers facing the DNA array, as expected in view of the strong electrostatic attraction between the CLs and DNA. It is possible that the final snapshot in Fig. 1 (B) is typical for an equilibrium distribution of free and complexed lipids, although long MC runs of pre-assembled complexes showed no evidence for escape of lipids from the complex.

One of the parameters which is believed to strongly influence the TE is the membrane charge density $\sigma_{M}=$ $e \phi_{c} / a_{\text {lipid }}[20]$. It was found in X-ray diffraction experiments that for isoelectric complexes the DNA interaxial spacing, $d_{\mathrm{DNA}}$, is related to $\sigma_{M}$ by $[11,14]$

$$
d_{\mathrm{DNA}}=\frac{\lambda_{D N A}}{\sigma_{M}}=\left(\frac{a_{\mathrm{lipid}} \lambda_{\mathrm{DNA}}}{e}\right) \frac{1}{\phi_{c}} .
$$

Measuring $d_{\mathrm{DNA}}$ and verifying this result can serve as a test for our model's ability to mimic the meso-scale behavior of CL-DNA complexes. We have therefore performed a set of MC simulations of pre-assembled isoelectric complexes consisting of 300 charged lipids, an array of five equally spaced DNA molecules of length $L_{y}=102 \AA$ (i.e., each carrying a total charge $-60 e$ ), and an additional number of neutral lipids. Simulations have been performed using the constant surface tension ensemble $(N, \gamma, T)$, with $\gamma=0[9,26]$. The area of the complex has been changed by allowing flexibility in $L_{x}$ (rescaling the $x$ coordinates of particles accordingly), keeping $L_{y}$ fixed. Attempts to change the area of the system were made, on average, twice every MC time unit. Two flat bilayers, each consisting of 150 cationic and $(2 N-150)$ neutral lipids, were initially set up on both side of the DNA array with the head groups in the inner (outer) monolayers at distance $0.5 \sigma+R_{\mathrm{DNA}}\left(5.5 \sigma+R_{\mathrm{DNA}}\right)$ from the complex mid-plane. Each monolayer consisted of $N$ lipids, with the CLs occupying the two inner monolayers while the outer monolayers consisting of neutral lipids only. This distribution of lipids remained unchanged over the course of the simulations (i.e., there was no diffusion of lipids between the monolayers on the time scale of the simulations). The fraction of charged lipids is defined as the number ratio of charged to neutral lipids in the inner monolayers, $\phi_{c}=150 / N$. The pre-assembled complexes were allowed to equilibrate for $5 \times 10^{5}$ steps prior to data collections over, at least, $2 \times 10^{6}$ additional steps. Membrane fluidity was verified by lateral diffusion of both neutral and charged lipids. The former diffuse faster than the latter.

The average spacing between adjacent DNA rods, $d_{\mathrm{DNA}}$, is plotted in Fig. 22 as a function of the inverse of the fraction of charged lipids, $1 / \phi_{c}$. The numerical data is in excellent agreement with the experimental results reported in Ref. [11]. The solid line is obtained

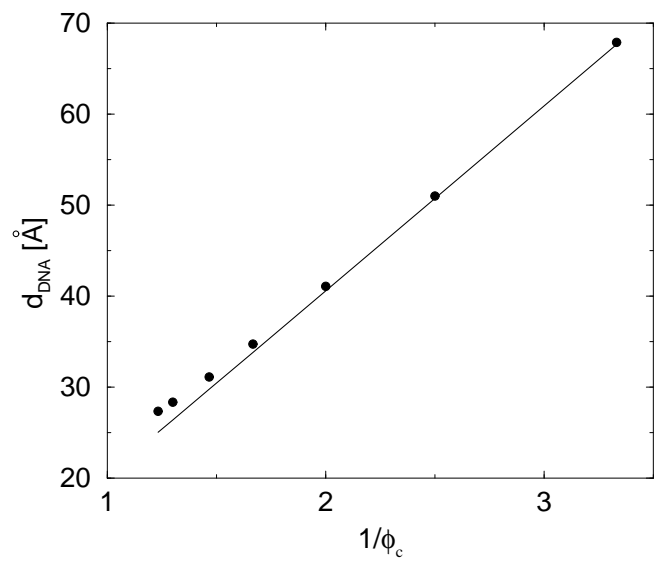

FIG. 2: Average DNA spacing, $d_{\text {DNA }}$ as a function of the inverse of the fraction of charged lipids $1 / \phi_{c}$. Circles - numerical results (the uncertainties in the data are smaller than the symbols); solid line - fit to Eq. (11) with $a_{\text {lipid }}=69 \AA^{2}$.

from Eq. (1) with $a_{\text {lipid }}=69 \AA^{2}$. The deviation from linear behavior at high charge densities arises from the increase in the area per lipid as $\phi_{c}$ (see Fig. 3 left $y$-axis, black circle symbols). Similar deviation is observed in the experiment 27]. The assumption underlying Eq. (11) is that the effective interactions between the DNA are repulsive and balanced by the elastic membrane forces. According to Hooke's law, the elastic stress acting on a membrane is related to $a_{\text {lipid }}$ and its equilibrium value $a_{\text {lipid }}^{0}$ by $\tau=K_{A}\left(a_{\text {lipid }}-a_{\text {lipid }}^{0}\right) / a_{\text {lipid }}^{0}$, where $K_{A}$ is the 2D stretching modulus, which for lipid bilayers is typically in the range $K_{A} \gtrsim 10^{2} \mathrm{ergs} / \mathrm{cm}^{2}$. At high charge densities, the electrostatic stress is sufficiently large to eliminate the membrane thermal undulations and increase $a_{\text {lipid }}$ [28]. In the present study, we find for complexes with $\phi_{c} \sim 0.85$ that the strain $\varepsilon \equiv\left(a_{\text {lipid }}-a_{\text {lipid }}^{0}\right) / a_{\text {lipid }}^{0} \sim 0.1$, which is the typical strain lipid membranes can withstand before rapture [29]. Membranes with higher $\phi_{c}$ have indeed been found to be susceptible to pore formation, as illustrated by the configuration in Fig. 1 (C) of a complex with $\phi_{c} \sim 0.9$. The loss of mechanical stability is also evident from the rapid decrease in the complex stretching modulus $K_{A}^{*}$ for $\phi_{c} \gtrsim 0.7$ (Fig. 3 right $y$-axis, red circle symbols), which has been extracted from the mean square fluctuation in $a_{\text {lipid }}: K_{A}^{*}=k_{B} T a_{\text {lipid }}^{0} /\left[N\left\langle\left(a_{\text {lipid }}-a_{\text {lipid }}^{0}\right)^{2}\right\rangle\right]$. The larger area fluctuations at high $\phi_{c}$ increase the probability of pore opening which in turn may lead to disassociation of the complex. We suggest that this may be the origin of the recently observed enhanced $\mathrm{TE}$ of lamellar CL-DNA complexes at high charge densities [20]. Transfection is viewed as a two-stage process: (1) cellular uptake via endocytosis, and (2) escape of the complex from the endosome, presumably through fusion of the lipids with the endosomal membrane and release of the DNA 


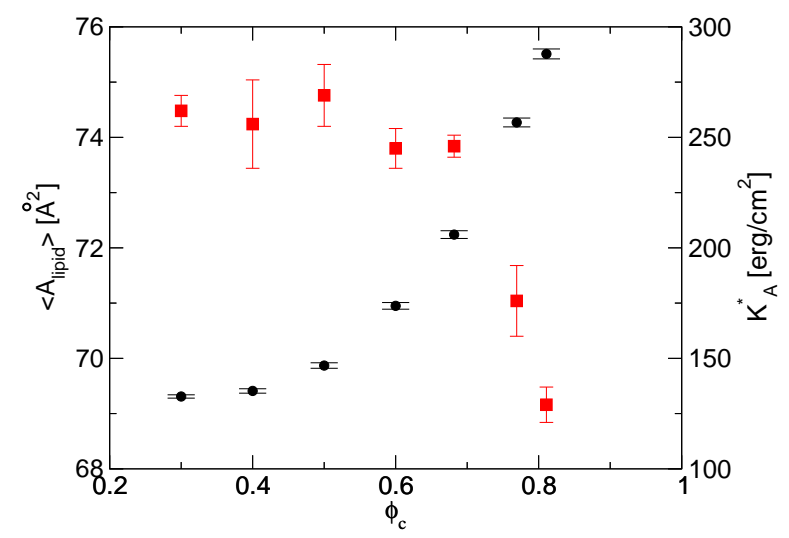

FIG. 3: (a) Left $y$-axis, black circle symbols - average area per lipid $a_{\text {lipid }}$ as a function of the fraction of charged lipids $\phi_{c}$. (b) Right $y$-axis, red square symbols - Stretching modulus $K_{A}^{*}$ of the complex as a function of $\phi_{c}$.

into the cytoplasm. TE of lamellar complexes is limited by the rate of the second stage and, hence, increases with the decrease of mechanical stability, i.e., with increase of charge density.

In summary, the structural-mechanical properties of CL-DNA complexes have been studied using a coarsegrained "water-free" model. Despite of the highly simplified representation of lipids and DNA, the model re- liably demonstrates self-assembly of both a charge neutral bilayer lipid membrane and a lipid membrane DNA complex. The model reproduces accurately the measured interaxial spacing $d_{\text {DNA }}$ over a wide range of charge densities. The wide applicability of the model should be attributed to the fact that the meso-scale behavior of the system is dominated by non-specific electrostatic and elastic interactions. At high charge densities we observe that the increasing electrostatic pressure exerted on the membranes leads to pore opening through which the DNA may be released from the complex. The DNA release to the cytoplasm is a necessary step for successful transfection and, thus, our results explain the enhancement of transfection efficiency at high concentrations of CLs. Given the consistency of agreement between our course-grained molecular approach and observed experimental features, we suggest that the presented model is an appropriate and promising tool for investigating the statistics and dynamics of lipid-DNA complexes on spatial and temporal scales relevant for biological and biomedical applications.

We are grateful to C. R. Safinya for many useful discussions. $\mathrm{OF}$ and $\mathrm{PP}$ acknowledge the support of the MRL Program of the National Science Foundation under Award No. DMR00- 80034 and NSF Grant No. DMR02037555 .
[1] M. Müller, K. Katsov, and M. Schick, J. Polymer Sci. B: Polymer Phys. 41, 1441 (2003), and Refs. therein.

[2] L. Saiz, S. Bandyopadhyay, and M. L. Klein, Bioscience Rep. 22, 151 (2002), and Refs. therein.

[3] R. Lipowsky and E. Sackmann Eds. Structure and Dynamics of Membranes (Elsevier, Amsterdam, 1995).

[4] S. O. Nielsen, C. F. Lopez, G. Srinivas, and M. L. Klein, J. Phys.: Condens. Matter 16, R481 (2004).

[5] E. Lindahl and O. Edholn, J. Chem. Phys. 113, 3882 (2000); S. J. Marrink and A. E. Mark, J. Phys. Chem. B 105, 6122 (2001).

[6] R. Goetz and R. Lipowsky, J. Chem. Phys. 108, 7397 (1998).

[7] H. Noguchi and M. Takasu, Phys. Rev. E 64, 041913 (2001).

[8] O. Farago, J. Chem. Phys. 119, 596 (2003); O. Farago and P. Pincus, J. Chem. Phys. 120, 2934 (2004).

[9] G. Brannigan, A. C. Tamboli, and F. L. H. Brown, J. Chem. Phys. 121, 3259 (2004).

[10] I. R. Cooke, K. Kremer, and M. Deserno, preprint cond-mat/0502418

[11] J. O. Rädler, I. Koltover, T. Salditt, and C. R. Safinya, Science 275, 810 (1997).

[12] J. O. Rädler, I. Koltover, T. Salditt, and C. R. Safinya, Science 281, 78 (1998).

[13] D. Harries, S. May, W. M. Gelbart, and A. Ben-Shaul, Biophys. J. 75, 159 (1998).

[14] C. R. Safinya, Curr. Opin. Struct. Biol. 11, 440 (2001).

[15] P. L. Felgner, Sci. Am. 276, 86 (1997).

[16] P. R. Clark and E. M. Hersh, Curr. Opin. Mol. Ther. 1,
158 (1999).

[17] S. Li and L. Huang, Gene Ther. 7, 31 (2000).

[18] H. Kamiya, H. Tsuchiya, J. Yamazaki, and H. Harashima, Adv. Drug Delivery Rev. 52, 153 (2001).

[19] H. F. Willard, Science 290, 1308 (2000).

[20] K. Evert et al., Curr. Med. Chem. 11, 133 (2004).

[21] S. May and A. Ben-Shaul, Curr. Med. Chem. 11, 151 (2004).

[22] R. Bruinsma, Euro. Phys. J. B4, 75 (1998).

[23] D. Harries, S. May, and A. Ben-Shaul, J. Phys. Chem. B 107, 3624 (2003).

[24] S. Bandyopadhyay, M. Tarek, and M. L. Klein, J. Phys. Chem. B 103, 10075 (1999).

[25] N. Grønbech-Jensen, G. Hummer, and K. M. Beardmore, Mol. Phys. 92, 941 (1997).

[26] An alternative scheme [O. Farago, in preparation (2005)] for sampling the $(N, \gamma, T)$ ensemble has been used in this work.

[27] The experimental results reported in 11] [see Fig. 4 (B)] show agreement with Eq. (1) at low charge densities (with a value of $a_{\text {lipid }}$ which is slightly different than the one defined here) and a similar deviation trend at high charge densities. Note that our Eq.(1) and the comparable one in Ref. 11] express the same relationship in different forms. See discussion in I. Koltover, T. Salditt, and C. R. Safinya, Biophys. J. 77, 915 (1999).

[28] A. W. C. Lau, and P. Pinucs, Eur. Phys. J. B 10, 175 (1999).

[29] D. Boal, Mechanics of the Cell (Cambridge Univ. Press, Cambridge, 2002). 